\title{
Pattern dystrophy of retinal pigment epithelium
}

\author{
Jacques Ramos Houly , $^{1, *}$ \\ ${ }^{1}$ Department of Ophthalmology, Federal University of Minas Gerais, Brazil \\ ${ }^{2}$ Fundação Hilton Rocha, Minas Gerais, Brazil
}

\section{Introduction}

Pattern dystrophies are a heterogeneous group of inherited retinal conditions that affect the retinal pigment epithelium (RPE) and are characterized by various patterns of pigment in the central macula. Pattern dystrophies are known to be caused by various mutations in the human retinal degeneration slow (RDS)/peripherin gene. The RDS/ peripherin gene encodes a photoreceptor-specific glycoprotein that may play a role in the development and maintenance of photoreceptor outer segment discs [1,2].

Mutations are associated with degeneration of photoreceptor cells and ultimately result in an accumulation of lipofuscin in the RPE [3,4].

Pattern dystrophies are characterized by bilateral deposits of yellow, orange or brown material at the level of the RPE. Based on the pattern of the pigment distribution pattern dystrophies have been classified into five prominent categories: adult-onset foveomacular vitelliform dystrophy, butterfly-shaped pigment dystrophy, reticular dystrophy, multifocal pattern dystrophy simulating Stargardt disease and fundus pulverulentus [5].

Patients with pattern dystrophy may be asymptomatic or have slight decrease of visual acuity with or without metamorphopsia. Diagnosis of this disease is based on the pattern of pigment deposition in the RPE. The age of onset is variable and symptoms, when present, tend to arise between the fourth and sixth decades of life. Fluorescein angiography can be helpful depending on the pattern and severity of disease. Results of electroretinogram (ERG) and electro-oculogram (EOG) maybe normal or abnormal, with mildy reduced electro-oculogram (EOG) consistent with diffuse RPE atrophy. Optical coherence tomography (OCT) may be useful in the analysis of retinal layers, description of the material deposits and evaluation of lesions at RPE level.

\section{Case description}

A 74-year-old male patient presented to a routine ophthalmologic examination at Fundação Hilton Rocha, Brazil. He did not have any complaints. A complete ophthalmic examination was performed, including refractometry with measurement of visual acuity, applanation tonometry, biomicroscopy and binocular indirect ophthalmoscopy. Best-corrected visual acuity (BCVA) was 20/30 in both eyes. Intraocular pressure was normal. Anterior segment examination findings were unremarkable. Fundoscopic examination revealed a normal optic disc with a small cup-to-disc ratio. Multiple yellowish fleck-like lesions and pigmentary changes were observed in the posterior pole in both eyes. The lesions had sub-retinal location and triradiate configuration (Figure 1). Fluorescein angiography of both eyes exhibited zones of hyperfluorescence that delineate areas of hypofluorescence. Furthermore, in the foveal center was detected hyperfluorescence in late frames (Figure 2). No leakage was seen in both eyes. Optical coherence tomography revealed attenuation of the foveal contour, presence of hyper-reflective material in the foveal center and drusen-like formations at the level of the outer photoreceptor segments (Figure 3). Electrophysiologic tests showed a normal full-field electroretinogram and abnormal electro-oculogram (EOG).

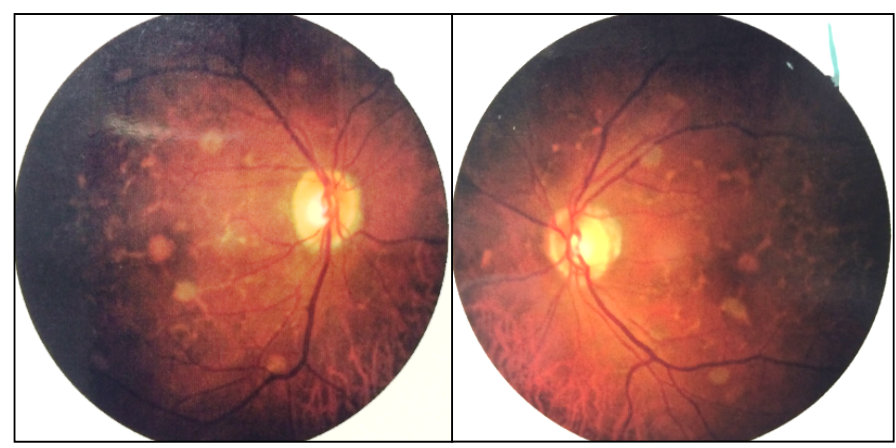

Figure 1. Fundus color photos show yellowish flecks, pigmentary changes and subfoveal lesion in the posterior pole (multifocal pattern dystrophy).

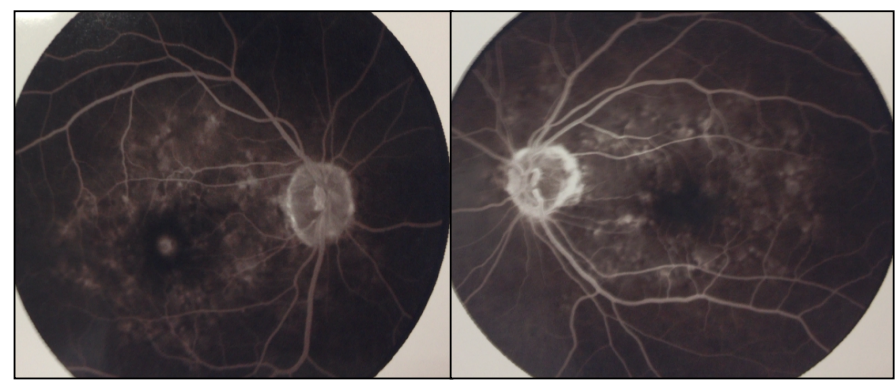

Figure 2. Fluorescein angiography depicts zones of hyperfluorescence that delineate areas of hypofluorescence. There is hyperfluorescence of the foveal center, especially in the right eye.

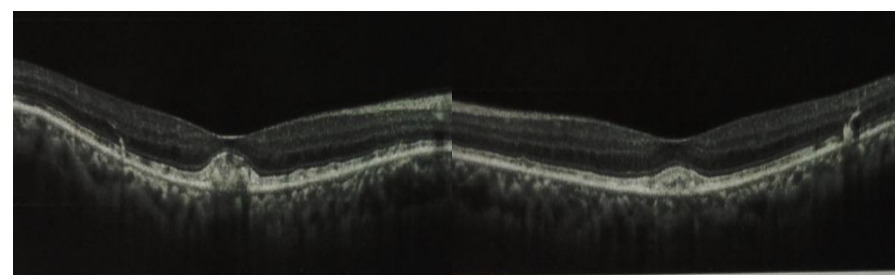

Figure 3. Optical coherence tomography images (horizontal scan) demonstrate subretinal hyper-reflective material in the foveal center and drusen-like formation at outer retinal layers.

Correspondence to: Jacques Ramos Houly, Department of Ophthalmology, Federal University of Minas Gerais, Brazil, E-mail: jacquesramos@ig.com.br

Received: May 06, 2017; Accepted: May 25, 2017; Published: May 29, 2017 


\section{Discussion}

Pattern dystrophy is a disease that affects the macula, has an insidious and progressive course and the characteristic pattern of pigment deposition determines the clinical classification of this disease. Pattern dystrophies are often associated with a relatively good visual acuity and visual disturbances and deficits typically progress very little, with vision remaining mostly intact throughout life. Although there are distinct patterns, some patients can change patterns or have different patterns in each eye [6]. Additionally, different types may occur in the same family. Due to these reasons, the clinical value of this classification is questionable.

This case report shows a patient with yellowish retinal lesions scattered throughout the posterior pole, resembling those found in Stargardt disease. Although genotypically similar to other pattern dystrophies, multifocal pattern dystrophy simulating Stargardt disease/fundus flavimaculatus is phenotypically singular. It can be distinguished from other autosomal dominant pattern dystrophies by its characteristic well demarcated, yellowish flecks with triradiate configuration $[3,7]$.

On fluorescein angiography, these flecks are hyperfluorescent. Clinical findings that may help to distinguish this pattern dystrophy from Stargardt disease are the autosomal dominant pattern of inheritance, the relatively late onset, the comparatively good and stable visual acuity and the absence of a "dark choroid" [8]. Genetic test of this patient is in progress and the result will help in the differential diagnosis of other diseases and genetic counseling.

Pattern dystrophy is caused by lipofuscin accumulation in the RPE layer of the retina [3]. In this case the SD-OCT was a useful tool in the assessment of the retinal layers, evaluation of the photoreceptors and at quantitative analysis of subretinal material and the electro-oculogram corroborated with the diagnostic hypothesis.

There is no cure for the pattern dystrophies, however most patients maintain reasonable good vision throughout their life without the need of any treatment. In this case the patient was instructed to use the Amsler grid and seek medical assistance in case of visual disturban

\section{References}

1. Arikawa K, Molday LL, Molday RS, Williams DS (1992) Localization of RDS Peripherin in the Disk Membranes of Cone and Rod Photoreceptors: Relationship to Disk Membrane Morphogenesis and Retinal Degeneration. J Cell Biol 116: 659-667. [Crossref]

2. Travis GH, Sutcliffe JG, Bok D (1991) The Retinal Degeneration Slow (RDS) Gene Product is a Photoreceptor Disc Membrane-associated Glycoprotein. Neuron 6: 61-70. [Crossref]

3. Zhang K, Garibaldi DC, Li Y, et al. (2002) Butterfly-Shaped Pattern Dystrophy: A Genetic, Clinical, and Histopathological report. Ophthalmic Mol Genet 120: 485-490. [Crossref]

4. Francis PJ1, Schultz DW, Gregory AM, Schain MB, Barra R, et al. (2005) Genetic and phenotypic heterogeneity in pattern dystrophy. Br J Ophthalmol 89: 1115-1119. [Crossref]

5. Gass JMD (1997). Stereoscopic Atlas of Macular Disease. Philadelphia, Elsevier.

6. Weleber RG1, Carr RE, Murphey WH, Sheffield VC, Stone EM (1993) Phenotypic variation including retinitis pigmentosa, pattern dystrophy, and fundus flavimaculatus in a single family with a deletion of codon 153 or 154 of the peripherin/RDS gene. Arch Ophthalmol 111: 1531-1542. [Crossref]

7. Boon CJ, van Schooneveld MJ, den Hollander AI, et al. (2007) Mutations in the peripherin/RDS gene are an important cause of multifocal pattern dystrophy simulating STGD1/fundus flavimaculatus. Br J Ophthalmol 91: 1504-1511. [Crossref]

8. Boon CJ1, den Hollander AI, Hoyng CB, Cremers FP, Klevering BJ, et al. (2008) The spectrum of retinal dystrophies caused by mutations in the peripherin/RDS gene. Prog Retin Eye Res 27: 213-235. [Crossref]

Copyright: (C2017 Houly JR. This is an open-access article distributed under the terms of the Creative Commons Attribution License, which permits unrestricted use, distribution, and reproduction in any medium, provided the original author and source are credited. 